\title{
TeV cosmic-ray electrons from millisecond pulsars
}

\author{
Shota Kisaka ${ }^{1}$ and Norita Kawanaka ${ }^{2}$ \\ ${ }^{1}$ Institute for Cosmic Ray Research, University of Tokyo, Kashiwa-no-ha 5-1-5, \\ Kashiwa-shi, Chiba, Japan \\ email: kisaka@icrr.u-tokyo.ac.jp \\ ${ }^{2}$ Racah Institute of Physics, Hebrew University of Jerusalem, Jerusalem 91904, Israel \\ email: norita@phys.huji.ac.il
}

\begin{abstract}
Recent $\gamma$-ray observations by the Fermi Gamma-Ray Space Telescope suggest that the $\gamma$-ray millisecond pulsar (MSP) population is separated into two subclasses with respect to pair multiplicity. Here, we calculate the cosmic-ray electron/positron spectra from MSPs. Based on the assumption of equipartition in the pulsar-wind region, the typical energy of electrons/positrons ejected by an MSP with pair multiplicity of the order of unity is $\sim 50 \mathrm{TeV}$. In this case, we find that a large peak in the 10-50 TeV energy range would be observed in the cosmic-ray electron/positron spectrum. Even if the fraction of pair-starved MSPs is 10\%, a large peak would be detectable with future missions such as CALET and CTA.
\end{abstract}

Keywords. stars: neutron, cosmic rays

\section{Introduction}

The Fermi Gamma-Ray Space Telescope has detected $\gamma$-ray pulsed emission from more than twenty millisecond pulsars (MSPs; Nolan et al. 2012), which have a rotation angular frequency $\Omega \sim 10^{3} \mathrm{~s}^{-1}$ and a stellar surface magnetic field $B_{\mathrm{s}} \sim 10^{8.5} \mathrm{G}$. The detection of $\mathrm{GeV}$ emission from a pulsar magnetosphere means that electrons and positrons are accelerated to more than $\sim \mathrm{TeV}$ by the electric field parallel to the magnetic field, which arises in a depleted region of the Goldreich-Julian (GJ) charge density (Goldreich \& Julian 1969).

Venter et al. (2009) fitted the pulse profiles of Fermi-detected MSPs with the geometries of the $\gamma$-ray emission regions predicted by different theoretical models. They found that the pulse profiles of the two MSPs show unusual behavior in the $\gamma$-ray light curves and could not be fitted by the geometry of either the outer-gap or slot-gap models. They proposed that these unusual light curves could be fitted by the pair-starved polar-cap model (Muslimov \& Harding 2004).

The high-energy electron/positron spectrum ejected from MSPs would be a useful probe for the existance of pair-starved MSPs. Since the lifetimes of MSPs are much longer than those of canonical pulsars $\left(>10^{10} \mathrm{yr}\right)$, there should be many more nearby active MSPs. Therefore MSPs could potentially contribute to the observed high-energy cosmic-ray electrons/positrons and will be detectable by next-generation experiments such as the Calorimetric Electron Telescope (CALET; Torii et al. 2008a) and Cherenkov Telescope Array (CTA; CTA Consortium 2010).

In this paper, we investigate the contribution of electrons/positrons ejected from MSPs to the observed cosmic-ray spectrum and show the possibility that the electrons/positrons from these MSPs will be detectable in future observations. 


\section{The Model}

In order to estimate the energy of electrons/positrons available in the wind region and their adiabatic and radiative cooling in the shocked region, we adopt the model of Kashiyama, Ioka \& Kawanaka (2011; hereafter KIK11). Although KIK11 considered the case of white dwarf pulsars, the situation is similar to the case of MSPs because they have a long lifetime and the supernova shock front has already decayed. From now on we set fiducial parameters of the MSP's surface magnetic field strength, angular frequency and radius as $B_{0}=10^{8.5} \mathrm{G}, \Omega=10^{3} \mathrm{~s}^{-1}$ and $R=10^{6} \mathrm{~cm}$, respectively.

We assume energy equipartition between the particles and the magnetic field, $\varepsilon_{e} N=$ $B^{2} / 8 \pi$, and the conservation of the particle number flux, $4 \pi r^{2} c N \sim$ constant, in the MSP wind region. Here, $N$ is the number density of electrons/positrons and $c$ is the speed of light. Using these assumptions, the typical energy of electrons/positrons $\varepsilon_{e}$ can be described as

$$
\varepsilon_{e} \sim 50 \kappa^{-1}\left(\frac{B_{0}}{10^{8.5} \mathrm{G}}\right)\left(\frac{\Omega}{10^{3} \mathrm{~s}^{-1}}\right)^{2}\left(\frac{R}{10^{6} \mathrm{~cm}}\right)^{3} \mathrm{TeV},
$$

where $\kappa$ is the multiplicity of electrons/positrons. Note that the typical energy depends on the pair multiplicity. We estimate the adiabatic and radiative cooling of electrons/positrons in the shocked region and can conclude that the energy loss of electrons/positrons in a pulsar-wind nebula is not very large (Kisaka \& Kawanaka 2012).

The observed electron/positron spectrum after propagation in the ISM is obtained by solving the diffusion equation

$$
\frac{\partial}{\partial t} f\left(t, r, \varepsilon_{e}\right)=D\left(\varepsilon_{e}\right) \nabla^{2} f+\frac{\partial}{\partial \varepsilon_{e}}\left(P\left(\varepsilon_{e}\right) f\right)+Q\left(t, r, \varepsilon_{e}\right)
$$

where $f\left(t, r, \varepsilon_{e}\right)$ is the energy distribution function of electrons/positrons, $D\left(\varepsilon_{e}\right)=D_{0}(1+$ $\left.\varepsilon_{e} / 3 \mathrm{GeV}\right)^{\delta}$ is the diffusion coefficient, $P\left(\varepsilon_{e}\right)$ is the cooling function of the electrons/ positrons, which takes into account synchrotron emission and inverse Compton scattering during the propagation, and $Q\left(t, \varepsilon_{e}, r\right)$ is the injection term. Here we adopt $D_{0}=5.8 \times$ $10^{28} \mathrm{~cm}^{2} \mathrm{~s}^{-1}, \delta=1 / 3$.

\section{Result and Discussion}

We calculate the cosmic-ray electron/positron spectra from pair-starved MSPs. We set the pair multiplicity $\kappa=1$, lifetime $\tau=5 \times 10^{10} \mathrm{yr}$, total energy $E_{\text {rot }}=10^{52} \mathrm{erg}$, local birth rate $R=3 \times 10^{-9} \mathrm{yr}^{-1} \mathrm{kpc}^{-2}$ and the fraction of energy lost due to synchrotron emission as 30 per cent for each MSP. We assume that each MSP has the same parameter values $\left(B_{0}=10^{8.5} \mathrm{G}, \Omega=10^{3} \mathrm{~s}^{-1}, R=10^{6} \mathrm{~cm}\right)$, because most MSPs have almost the same spin-down luminosity. For the injection distribution function, we assume a mono-energetic distribution equation with energy $\varepsilon_{e}=50 \mathrm{TeV}$.

In Fig. 1, the electron/positron flux from multiple pair-starved MSPs is shown. It is very interesting that there is a large peak at 10-50 TeV energy range. We should detect the electron/positron flux with near-future missions such as CALET and CTA.

It was considered that the number of astrophysical sources contributing to the energy range above several $\mathrm{TeV}$ is quite small, according to the birth rate of supernovae and canonical pulsars in the vicinity of Earth (Kobayashi et al. 2004; Kawanaka, Ioka \& Nojiri 2010; Kawanaka et al. 2011). However, we find that it is possible for multiple pair-starved MSPs to contribute to the 10-TeV energy range in the electron/positron spectrum. Therefore, if the anisotropy of observed electrons/positrons is weak in that range, we suggest that pair-starved MSPs may contribute to the spectrum significantly. 


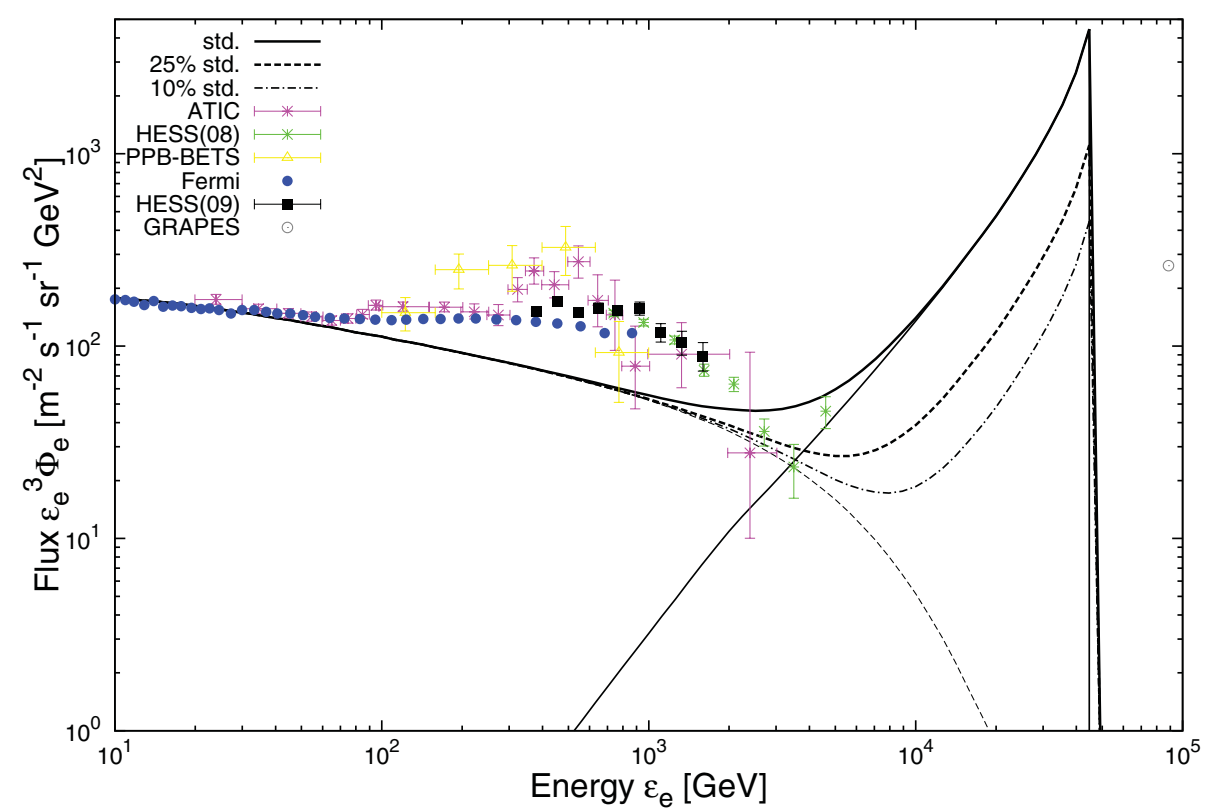

Figure 1. Electron/positron spectrum predicted from MSPs with a fraction of pair-starved MSPs of $100 \%$ (thin solid line) and its sum (thick solid line) together with the background (thin-dashed line). The injection distribution function is a mono-energetic distribution with energy $\varepsilon_{e}=50 \mathrm{TeV}$ and multiplicity $\kappa=1$. Data points correspond to measurements of ATIC (boxes, purple; Chang et al. 2008), HESS (asterisks, light green and black; Aharonian et al. 2008, 2009), PPB-BETS (triangles, yellow; Torii et al. 2008b), Fermi (shaded circles, blue; Ackermann et al. 2010) and GRAPES (black empty circles: Kistler \& Yüksel 2009). We also show the total spectra from pair-starved MSPs with different fractions: $25 \%$ (thick-dashed line) and $10 \%$ (dot-dashed line). We assume that the lifetime $\tau=5 \times 10^{10} \mathrm{yr}$, total energy $E_{\mathrm{rot}}=10^{52}$ erg, local birth rate $R=3 \times 10^{-9} \mathrm{yr}^{-1} \mathrm{kpc}^{-2}$ and the fraction of energy loss is $30 \%$ (Kisaka \& Kawanaka 2012)

\section{References}

Ackermann M., et al. 2010, Phys. Rev. D, 82, 092004

Aharonian F. A., et al. 2008, Phys. Rev. Lett., 101, 261104

Aharonian F. A., et al. 2009, A\&\&A, 508, 561

Chang J., et al. 2008, Nature, 456, 362

CTA Consortium 2010, preprint, (arXiv:1008.3703)

Goldreich P. \& Julian W. H. 1969, ApJ, 157, 869

Kashiyama K., Ioka K., \& Kawanaka, N. 2011, Phys. Rev. D, 83, 023002

Kawanaka N., Ioka K., \& Nojiri M. M. 2010, ApJ, 710, 958

Kawanaka N., Ioka K., Ohira Y., \& Kashiyama K. 2011, ApJ, 729, 93

Kisaka S. \& Kawanaka N. 2012, MNRAS, 421, 3543

Kistler M. D. \& Yüksel H. 2009, preprint, (arXiv:0912.0264)

Kobayashi T., Komori Y., Yoshida K., \& Nishinuma J. 2004, ApJ, 601, 340

Muslimov A. G. \& Harding A. K. 2004b, ApJ, 617, 471

Nolan, P. L., et al. 2012, ApJS, 199, 31

Torii S., et al. 2008a, J. Phys. Conf. Ser., 120, 062020

Torii S., et al. 2008b, preprint, (arXiv:0809.0760)

Venter C., Harding A. K., \& Guillemot L. 2009, ApJ, 707, 800 